\title{
Effects of paternal age on human embryo development in in vitro fertilization with preimplantation genetic screening
}

\author{
Min Kyoung Kim ${ }^{1}$, Jae Kyun Park ${ }^{1,2}$, Yunmi Jeon' ${ }^{1}$, Su Hee Seok', Eun Mi Chang ${ }^{1}$, Woo Sik Lee ${ }^{1}$ \\ 'Department of Obstetrics and Gynecology, Fertility Center of CHA Gangnam Medical Center, CHA University School of Medicine, Seoul; ${ }^{2}$ Department \\ of Biomedical Science, CHA University, Seongnam, Korea
}

\begin{abstract}
Objective: As paternal age increases, the quality of sperm decreases due to increased DNA fragmentation and aneuploidy. Higher levels of structural chromosomal aberrations in the gametes ultimately decrease both the morphologic quality of embryos and the pregnancy rate. In this study, we investigated whether paternal age affected the euploidy rate.

Methods: This study was performed using the medical records of patients who underwent in vitro fertilization (IVF) procedures with preimplantation genetic screening (PGS) from January 2016 to August 2017 at a single center. Based on their morphological grade, embryos were categorized as good- or poor-quality blastocysts. The effects of paternal age were elucidated by adjusting for maternal age.

Results: Among the 571 total blastocysts, 219 euploid blastocysts were analyzed by PGS (38.4\%). When the study population was divided into four groups according to both maternal and paternal age, significant differences were only noted between groups that differed by maternal age (group 1 vs. $3, p=0.031$; group 2 vs. 4, $p=0.027$ ). Further analysis revealed no significant differences in the euploidy rate among the groups according to the morphological grade of the embryos.

Conclusion: Paternal age did not have a significant impact on euploidy rates when PGS was performed. An additional study with a larger sample size is needed to clarify the effects of advanced paternal age on IVF outcomes.
\end{abstract}

Keywords: Embryo quality; In vitro fertilization; Paternal age; Preimplantation genetic screening

\section{Introduction}

Couples delay childbearing due to higher education, increased participation of women in the workforce, and improved contraception. Assisted reproductive technology (ART) has also extended the reproductive window. Many studies have shown that maternal age is a major factor that affects the success rate of ART. In older women un-

Received: Nov 14, 2018 · Revised: Jan 9, 2019. Accepted: Jan 25, 2019 Corresponding author:Woo Sik Lee

Department of Obstetrics and Gynecology, Fertility Center of CHA Gangnam Medical Center, CHA University School of Medicine, 569 Nonhyeon-ro, Gangnam-gu, Seoul 06125, Korea

Tel: +82-2-3468-3000 Fax:+82-2-3468-2609 E-mail:wooslee@cha.ac.kr

This is an Open Access article distributed under the terms of the Creative Commons Attribution Non-Commercial License (http://creativecommons.org/licenses/by-nc/4.0/) which permits unrestricted non-commercial use, distribution, and reproduction in any medium, provided the original work is properly cited. dergoing in vitro fertilization (IVF), the number of retrieved oocytes [1], fertilization rate [2], and implantation rate [3] are lower than in younger patients. Paternal age is also increasing [4], but the effect of paternal age on IVF has been studied much less, and the results are conflicting.

A systematic review of 10 studies on the association between paternal age and ART outcomes did not show clear correlations between advanced paternal age and the rates of fertilization, implantation, pregnancy, miscarriage, and live birth [5]. Another recent metaanalysis investigated 12 studies on the influence of paternal age on oocyte donation outcomes to exclude bias due to the factor of maternal age [6]. Similarly, no statistically significant correlation between advanced paternal age and clinical outcomes was found.

In contrast, some studies reported negative effects of advanced paternal age on ART outcomes. For instance, 237 oocyte donation cy- 
cles were reviewed and the implantation rate significantly declined with increasing paternal age [7]. Each 5-year increase in paternal age was associated with $26 \%$ lower odds of live birth. An observational study comparing sperm genomic decay in young and advanced paternal age groups showed increased DNA fragmentation, chromatin decondensation, and sperm aneuploidy rates in older men [8]. IVF outcomes such as the decision to perform embryo transfer, the clinical pregnancy rate, and the miscarriage rate were also negatively affected. Therefore, this study aimed to investigate the effect of paternal age on euploidy in embryos and clinical outcomes after IVF and preimplantation genetic screening (PGS).

\section{METHODS}

\section{Patients}

This was a retrospective study reviewing the medical records of infertile patients undergoing IVF procedures from January 2016 to August 2017 at the Fertility Center of CHA Gangnam Medical Center. Patients who responded normally to ovarian stimulation were recruited. The normal responders were selected according to age, antiMüllerian hormone (AMH) levels, follicle-stimulating hormone (FSH) levels, and number of aspirated oocytes. PGS at the blastocyst stage was offered to infertile patients of advanced age ( $\geq 35$ years), and/or with $\geq 3$ unexplained recurrent pregnancy losses and/or $\geq 3$ recurrent implantation failures. Patients who did not undergo PGS and those who underwent 3-day embryo transfer were excluded from the study population. Study participants were grouped according to age: women aged $<35$ years and men aged $\leq 38$ years (group 1 ), women aged $<35$ years and men aged $>38$ years (group 2), women aged $\geq 35$ years and men aged $\leq 38$ years (group 3 ), and women aged $\geq 35$ years and men aged $>38$ years (group 4 ). The paternal age cutoff of 38 years was defined according to the most equilibrated distribution of cases in both age groups and on the basis of another study [9].

\section{Clinical protocols}

Gonadotropin-releasing hormone (GnRH) antagonist and agonist protocols were mostly used in this study. The following medications were used in these protocols: recombinant FSH (Gonal-F, Merck, Darmstadt, Germany; Pergoveris, Merck), a GnRH antagonist (Cetrotide, Merck), and a GnRH agonist (Lorelin; Dongkook Pharmaceutical, Seoul, Korea). Recombinant FSH doses were formulated according to the patient's antral follicular count, AMH level, and previous response to stimulation.

Transvaginal ultrasound was performed to monitor the follicular response to stimulation, and gonadotropin doses were adjusted accordingly. Final oocyte maturation was triggered with recombinant human chorionic gonadotropin (hCG) (Ovidrel, Merck) when there were $\geq 2$ follicles with a mean diameter $\geq 17 \mathrm{~mm}$. Ultrasound-guided oocyte retrieval under conscious sedation was performed 34-36 hours after the trigger. Luteal phase support was provided with progesterone vaginal suppositories (Utrogestan; Han Wha Pharma, Seoul, Korea) starting on the day of ovum pick-up. The $\beta$-hCG level was checked 10-12 days after embryo transfer.

\section{Embryo culture, grading, and biopsy procedures}

Intracytoplasmic sperm injection (ICSI) was used to fertilize all the embryos from fresh cycles. Of the thawed embryo transfer (TET) cycles, ICSI was used in 57\%, half-ICSI (ICSI and conventional IVF combined) in $15.9 \%$, and conventional IVF in $27.1 \%$, since for some patients, clinicians decided to perform PGS after embryo cryopreservation. For ICSI, the collected semen was liquefied for 30 minutes on a $37^{\circ} \mathrm{C}$ hot plate, after which sperm motility was evaluated [10]. Semen analysis was done, and semen samples with a normal sperm count and motility were washed two times with 10\% SPS including Ham's F-10 media by centrifuge. Motile spermatozoa were collected using the swim-up method. The general condition of each spermatozoon was thoroughly checked by first inspecting its general motility and morphology (head, midpiece, and tail), and then its head shape was evaluated. A spermatozoon was considered to have normal morphology when its head length was $5-7 \mu \mathrm{m}$, its width was $2-3 \mu \mathrm{m}$, and the acrosome distribution was more than 70\% [11]. After selecting normal sperm, ICSI was performed under microscopy with $\times 200$ magnification. In total, ICSI was used in $73.7 \%$ of cycles, half-ICSI in $9.7 \%$, and conventional IVF in $16.6 \%$. Fertilization was confirmed when the two-pronucleus stage was observed after 16-20 hours. During the cleavage stage, embryos were cultured in cleavage medium (Cook, Eight Mile Plains, Australia), and then transferred to blastocyst medium (Cook) on day 3.

Embryos were cultured in incubators (HERAcell 240i; Thermo Fisher Scientific, Waltham, MA, USA) under $5 \% \mathrm{O}_{2}$ and $6 \% \mathrm{CO}_{2}$ at $37^{\circ} \mathrm{C}$. Oil drop culture was done using a four-well dish (Nunc, Thermo Fisher Scientific). In 20- $\mu \mathrm{L}$ drops, a single round of embryo culture was carried out to facilitate the observation of embryonic development. Light paraffin oil (Ovoil; Vitrolife, Göteborg, Sweden) was dropped onto the media to prevent it from drying or undergoing a rapid $\mathrm{pH}$ change.

Morphological grading of the embryos after culture was done on day 5 (Figure 1). Embryo quality on day 5 was assessed according to the criteria of Gardner and Schoolcraft [12]. Good-quality embryos on day 5 were defined as those with a blastocoel equal to or greater than half the volume of the embryo and a good inner cell mass and trophectoderm (TE). Based on the morphological grade, the embryos were categorized as good-quality blastocysts (excellent, good, or av- 

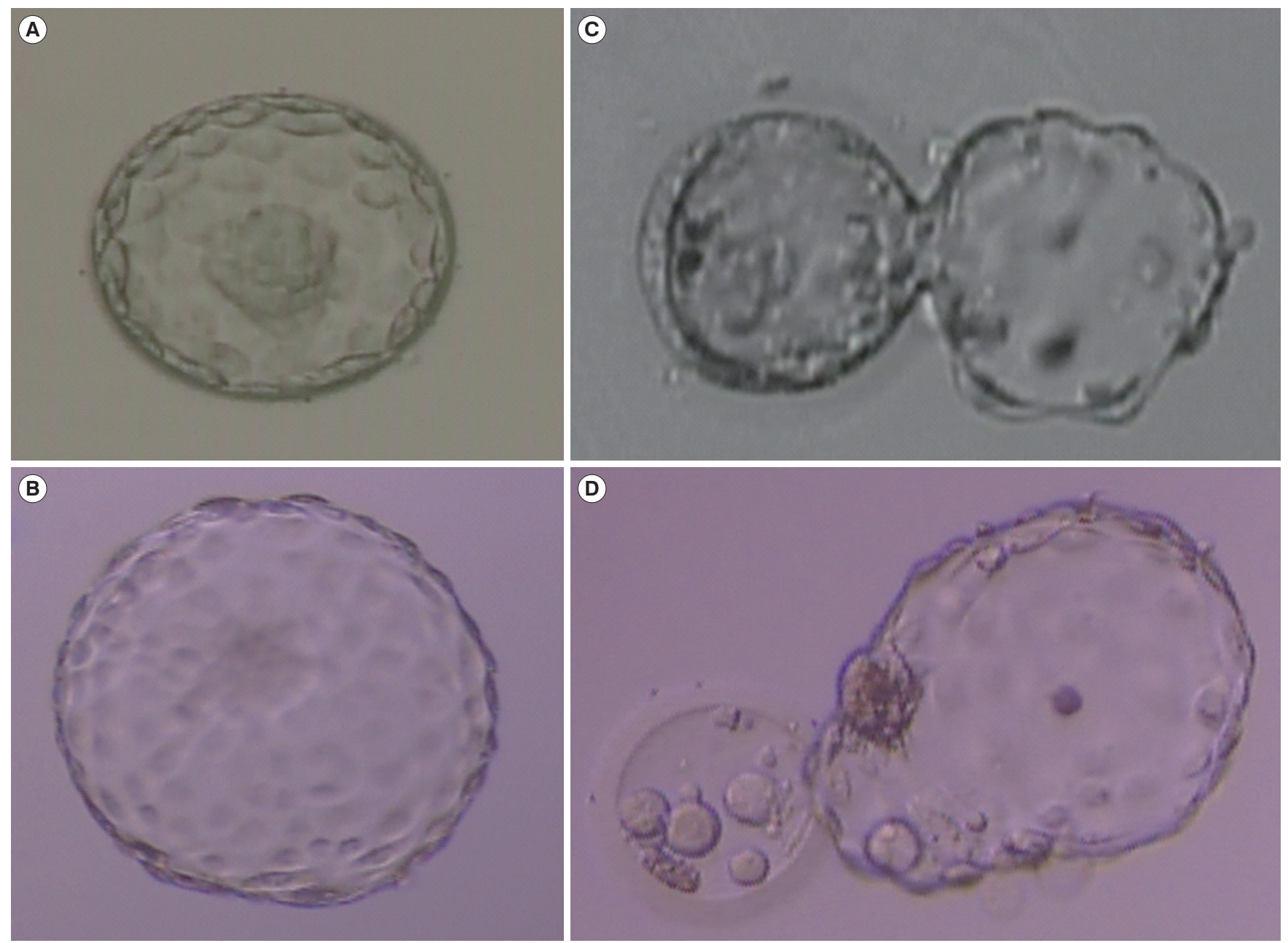

Figure 1. Examples of evaluations of human blastocyst morphology. (A) High-quality expanded blastocyst: type A ICM, type A TE. (B) Highquality hatched blastocyst: type A ICM, type A TE. (C) Low-quality hatching blastocyst: type C ICM, type C TE. (D) Low-quality hatched blastocyst: type C ICM, type CTE. (A-D) × 100 magnification. ICM, inner cell mass; TE, trophectoderm.

erage) and poor-quality blastocysts.

All embryos were biopsied on day 5, during the blastocyst stage. TE biopsy was done from blastocysts that were cultured after conventional IVF or ICSI using a pair of micromanipulators (NT- 88; Narishige, Tokyo, Japan) with a microscope (TE-2000; Nikon, Tokyo, Japan). The culture medium consisted of G-PGD (Vitrolife) with 5\% human albumin solution (Vitrolife). For the TE biopsy, the embryos were immobilized with a holding pipette (left side), and the zona pellucida was perforated using laser pulses with a ZILOS-tk noncontact laser (Hamilton Thorne Biosciences, Beverly, MA, USA). Using the right-side biopsy pipette, gentle suction of the TE cells was performed through the zona pellucida perforation. The cells were eventually separated out of the zona pellucida. The biopsied TE cells were washed with Dulbecco's phosphate-buffered saline (with $\mathrm{Ca}^{2+}$ and $\mathrm{Mg}^{2+}$ removed) and placed in RNase/DNase-free 0.2-mL polymerase chain reaction (PCR) tubes for referral to MG-MED Inc. (Seoul, Korea) for PGS. The bi- opsied blastocysts were then cultured in sterile oil-covered media under $6 \% \mathrm{CO}_{2}$ and $5 \% \mathrm{O}_{2}$ at $37^{\circ} \mathrm{C}$, and observations were made at 24 to 48-hour intervals to check the development of the embryos.

Embryo transfer was performed on day 6 under transabdominal sonographic guidance. Surplus embryos that were left after transfer were cultured, and the good-quality blastocysts on day 5 underwent PGS. Only normal euploid embryos were cryopreserved on day 6.

\section{PGS (array comparative genomic hybridization) protocol}

The biopsied cells were washed and collected in a PCR tube with 2.5 $\mu \mathrm{L}$ of PBS. The DNA from the biopsied cells was extracted and amplified using a MG Flex single-cell whole genome amplification kit (MG Flex; MGmed, Seoul, Korea) following the manufacturer's protocol. Approximately $3 \mu \mathrm{g}$ of amplified DNA was used in the array comparative genomic hybridization ( $\mathrm{aCGH}$ ) experiments (MGmed). Briefly, the amplified DNA was labeled with Cy-3 and Cy-5 deoxycytidine tri- 
phosphate for 3 hours using a random priming method. The labeled DNA was purified, dissolved in hybridization buffer, and hybridized overnight. The slides were washed several times and dried. Images of the slides were acquired with a GenePix 4000B dual-laser scanner (Axon Instruments, Union City, CA, USA) and analyzed with MGViewer analysis software (MGmed).

\section{Outcome measures and statistical analysis}

Clinical pregnancy was defined as the presence of at least one gestational sac and a fetal heartbeat at week 7 after transfer. Women over 16 weeks of gestation were defined as having an ongoing pregnancy. Continuous data are expressed as mean \pm standard deviation unless stated otherwise, and categorical variables are presented as absolute numbers and percentage. The data were analyzed using the Student $t$-test and the chi-square test. The analysis of clinical outcomes between different paternal age groups was done using the Mantel-Haenszel chi-square test to adjust for maternal age differences. All statistical analyses were performed using IBM SPSS ver. 25.0 (IBM Corp., Armonk, NY, USA). The $p$-values $<0.05$ were considered to indicate statistical significance.

Table 1. IVF cycle characteristics and outcomes

\begin{tabular}{|c|c|}
\hline Variable & Value \\
\hline No. of cycles & 175 \\
\hline Overall average female age (yr) & $36.0 \pm 3.8$ \\
\hline Overall average paternal age (yr) & $38.8 \pm 5.0$ \\
\hline $\begin{array}{l}\text { Overall average difference between paternal } \\
\text { and maternal age (yr) }\end{array}$ & $2.9 \pm 3.6$ \\
\hline $\mathrm{BMI}\left(\mathrm{kg} / \mathrm{m}^{2}\right)$ & $21.2 \pm 3.2$ \\
\hline No. of previous IVF attempts & $2.4 \pm 0.7$ \\
\hline AMH concentration (ng/mL) & $4.8 \pm 4.0$ \\
\hline Antral follicle count & $14.7 \pm 8.4$ \\
\hline Basal $E_{2}$ concentration $(\mathrm{pg} / \mathrm{mL})$ & $46.8 \pm 28.6$ \\
\hline Basal LH concentration (mlU/mL) & $5.3 \pm 2.6$ \\
\hline Basal FSH concentration (mlU/mL) & $6.6 \pm 2.3$ \\
\hline $\mathrm{E}_{2}$ concentration on $\mathrm{hCG}$ day $(\mathrm{pg} / \mathrm{mL})$ & $3,591.4 \pm 2,272.5$ \\
\hline LH concentration on $\mathrm{hCG}$ day (mlU/mL) & $3.1 \pm 3.2$ \\
\hline Progesterone concentration on $\mathrm{hCG}$ day $(\mathrm{ng} / \mathrm{mL})$ & $1.0 \pm 0.6$ \\
\hline $\begin{array}{l}\text { Overall average endometrial thickness on } \\
\text { hCG day }(\mathrm{cm})\end{array}$ & $1.0 \pm 0.2$ \\
\hline No. of total oocytes & 2,959 \\
\hline Fertilized oocytes & $71.0(2,100 / 2,959)$ \\
\hline No. of blastocysts analyzed for PGS & 571 \\
\hline Euploid blastocysts & $38.4(219 / 571)$ \\
\hline
\end{tabular}

Values are presented as mean \pm standard deviation or percent (number). IVF, in vitro fertilization; $\mathrm{BMI}$, body mass index; $\mathrm{AMH}$, anti-Müllerian hormone; $\mathrm{E}_{2}$, estradiol; $\mathrm{LH}$, luteinizing hormone; $\mathrm{FSH}$, follicle-stimulating hormone; $\mathrm{hCG}$, human chorionic gonadotropin; PGS, preimplantation genetic screening.

\section{Ethics statement}

The study was approved by the Institutional Review Board of CHA Gangnam Medical Center (IRB No. GCl-17-40). Consent to participate was waived due to its retrospective nature and only medical records were used in this analysis.

\section{Results}

A total of 175 cycles were included: 68 were fresh cycles and 107 were cryopreserved to be used in TET afterwards. Altogether, embryo transfer was performed in 107 cycles. Among the 571 total blastocysts, 219 euploid blastocysts were analyzed by PGS (38.4\%) and the IVF cycle characteristics are shown in Table 1. Table 2 describes the outcomes of the cycles in which embryo transfer was performed. A total of 140 euploid embryos were transferred and $46.4 \%$ succeeded in implantation; furthermore, $50.5 \%$ of the 107 transferred cycles resulted in clinical pregnancy, and $21.5 \%$ of the implanted embryos aborted.

Table 3 shows the characteristics and IVF outcomes of the euploid embryo transfers by dividing them into four groups according to maternal and paternal age. In groups 1 and 2, with a maternal age below 35 , the euploidy rates were $47.6 \%$ and $47.2 \%$, respectively. In groups 3 and 4, with advanced maternal age, the euploidy rates were $35.7 \%$ and $28.6 \%$, respectively. Correlations were analyzed based on this division of the study population into four groups (Figure 2A). Significant differences were only found between groups 1 and 3 and between groups 2 and 4 (group 1 vs. 3: $p=0.031$; group 2 vs. 4 : $p=0.027$ ), demonstrating that advanced paternal age did not have a major influence on the embryo euploidy rate, but advanced maternal age did have a significant impact. This supports the proposal that

Table 2. Embryo and clinical outcomes

\begin{tabular}{lc}
\hline Variable & Value \\
\hline No. of cycles & 175 \\
All cryopreservation & 26 \\
No ET & 42 \\
ET & 107 \\
No. of euploid blastocysts & 219 \\
Cryopreservation & 57 \\
Refreezing & 5 \\
Discard & 17 \\
ET & 140 \\
Clinical pregnancy rate & $50.5(54 / 107)$ \\
Implantation rate & $46.4(65 / 140)$ \\
Abortion rate & $21.5(14 / 65)$ \\
Twin gestation rate & $8.4(9 / 107)$
\end{tabular}

Values are presented as percent (number) unless otherwise indicated. $\mathrm{ET}$, embryo transfer. 
maternal age is more closely correlated than paternal age with human embryo development.

Further analysis was done to determine whether paternal age had an effect on the euploidy rate depending on the morphological grade of the blastocysts. The euploidy rates of good- and poor-grade blastocysts were $65.8 \%$ and $47.5 \%$ in group $1 ; 63.6 \%$ and $40 \%$ in group 2; $44.1 \%$ and $28.6 \%$ in group 3; and $29.3 \%$ and $27.8 \%$ in group 4 , respectively (Figure $2 \mathrm{~B}$ ). Although the proportions seemed to decrease as age increased, comparisons among the groups did not show any significant differences (group 1 vs. $2, p=0.584$; group 3 vs. $4, p=0.284$; group 1 vs. $3, p=0.747$; group 2 vs. $4, p=0.190$ ). Thus, no correlation was found between paternal age and the euploidy rate

Table 3. IVF cycle characteristics and outcomes with subjects divided into four groups by maternal and paternal age

\begin{tabular}{|c|c|c|c|c|c|}
\hline Variable & Group 1 & Group 2 & Group 3 & Group 4 & $p$-value \\
\hline No. of cycles & 58 & 12 & 37 & 68 & \\
\hline Average maternal age (yr) & $32.0 \pm 1.8$ & $33.42 \pm 0.8$ & $36.9 \pm 1.9$ & $39.3 \pm 2.4$ & $<0.001$ \\
\hline Average paternal age (yr) & $34.5 \pm 2.4$ & $40.83 \pm 2.4$ & $36.6 \pm 1.9$ & $43.4 \pm 4.0$ & $<0.001$ \\
\hline Average difference: paternal-maternal age (yr) & $2.6 \pm 2.2$ & $7.42 \pm 2.5$ & $-0.3 \pm 2.7$ & $2.9 \pm 2.1$ & $<0.001$ \\
\hline $\operatorname{BMl}\left(\mathrm{kg} / \mathrm{m}^{2}\right)$ & $21.1 \pm 3.6$ & $22.48 \pm 4.2$ & $21.1 \pm 2.6$ & $21.1 \pm 3.1$ & 0.55 \\
\hline No. of previous IVF attempts & $2.0 \pm 1.3$ & $2.83 \pm 5.7$ & $2.1 \pm 1.4$ & $2.9 \pm 2.1$ & 0.11 \\
\hline AMH concentration $(\mathrm{ng} / \mathrm{mL})$ & $4.7 \pm 3.3$ & $4.31 \pm 2.5$ & $4.1 \pm 3.4$ & $5.3 \pm 4.9$ & 0.80 \\
\hline Antral follicle count & $16.3 \pm 7.4$ & $13.83 \pm 11.0$ & $13.4 \pm 6.5$ & $14.2 \pm 9.5$ & 0.18 \\
\hline Basal $\mathrm{E}_{2}$ concentration (pg/mL) & $44.3 \pm 24.8$ & $35.37 \pm 13.7$ & $43.4 \pm 17.3$ & $52.8 \pm 36.9$ & 0.22 \\
\hline Basal LH concentration (mIU/mL) & $5.0 \pm 2.0$ & $6.68 \pm 3.5$ & $5.2 \pm 2.6$ & $5.3 \pm 3.0$ & 0.30 \\
\hline Basal FSH concentration (mlU/mL) & $6.5 \pm 2.4$ & $6.67 \pm 1.3$ & $6.8 \pm 2.2$ & $6.4 \pm 2.4$ & 0.69 \\
\hline $\mathrm{E}_{2}$ concentration on $\mathrm{hCG}$ day $(\mathrm{pg} / \mathrm{mL})$ & $3,614.2 \pm 1,802.8$ & $4,416.1 \pm 2,502.6$ & $3,332.3 \pm 1,993.6$ & $3,566.8 \pm 2,714.5$ & 0.67 \\
\hline LH concentration on hCG day (mlU/mL) & $2.9 \pm 2.4$ & $3.84 \pm 3.3$ & $2.3 \pm 2.3$ & $3.7 \pm 4.2$ & 0.38 \\
\hline Progesterone concentration on hCG day (ng/mL) & $1.0 \pm 0.5$ & $1.12 \pm 0.6$ & $0.9 \pm 0.7$ & $1.0 \pm 0.5$ & 0.95 \\
\hline Average endometrial thickness on hCG day (cm) & $1.0 \pm 0.2$ & $1.05 \pm 0.3$ & $1.0 \pm 0.2$ & $0.9 \pm 0.2$ & 0.07 \\
\hline No. of total oocytes & $18.6 \pm 10.9$ & $18.5 \pm 8.6$ & $15.9 \pm 7.4$ & $15.7 \pm 11.0$ & 0.29 \\
\hline Fertilized oocyte (\%) & 67.7 & 70.7 & 71 & 74.3 & 0.39 \\
\hline No. of blastocysts analyzed for PGS & 210 & 36 & 129 & 196 & - \\
\hline Euploid blastocyst & $47.6(100 / 210)$ & $47.2(17 / 36)$ & $35.7(46 / 129)$ & $28.6(56 / 196)$ & 0.001 \\
\hline Abortion rate & $2.4(1 / 41)$ & $0(0 / 8)$ & $20.0(4 / 20)$ & $18.4(7 / 38)$ & - \\
\hline Clinical pregnancy rate & $51.2(21 / 41)$ & $37.5(3 / 8)$ & $65.0(13 / 20)$ & $44.7(17 / 38)$ & 0.44 \\
\hline
\end{tabular}

Values are presented as mean \pm standard deviation or percent (number) unless otherwise indicated. Group 1: maternal age (MA) $<35$ year, paternal age (PA) $\leq 38$ year; Group 2: MA < 35 year, $\mathrm{PA}>38$ year; Group 3: $\mathrm{MA} \geq 35$ year, $\mathrm{PA} \leq 38$ year; Group 4: $\mathrm{MA} \geq 35$ year, $\mathrm{PA}>38$ year.

IVF, in vitro fertilization; $\mathrm{BMI}$, body mass index; $\mathrm{AMH}$, anti-Müllerian hormone; $\mathrm{E}_{2}$, estradiol; $\mathrm{LH}$, luteinizing hormone; FSH, follicle-stimulating hormone; $\mathrm{hCG}$, human chorionic gonadotropin; PGS, preimplantation genetic screening.
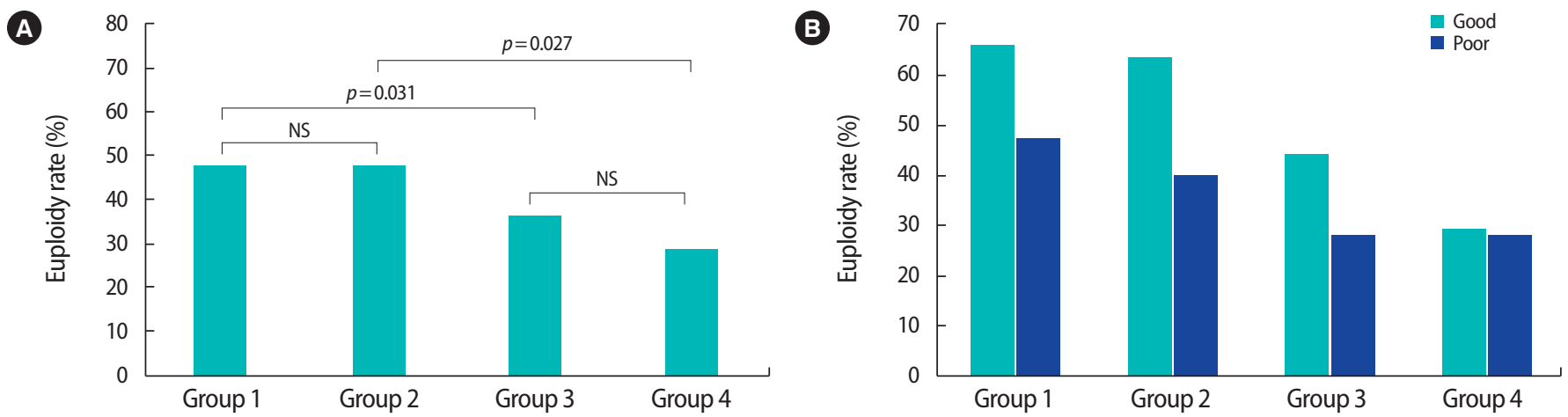

Figure 2. Embryo euploidy rates by maternal and paternal age and morphological grade. (A) Comparisons of the embryo euploidy rate with the study population divided into four groups by maternal and paternal age. Only the comparisons among groups 1 vs. 3 and 2 vs. 4 showed significant differences, demonstrating that advanced paternal age did not influence the embryo euploidy rate, while advanced maternal age had a significant impact. Group 1: maternal age (MA) < 35 year, paternal age $(\mathrm{PA}) \leq 38$ year; Group 2: MA <35 year, PA > 38 year; Group 3: MA $\geq 35$ year, PA $\leq 38$ year; Group 4: MA $\geq 35$ year, PA $>38$ year. (B) The euploidy rates of blastocysts with different morphological grades showed no significant differences among the four groups. NS, not significant. 


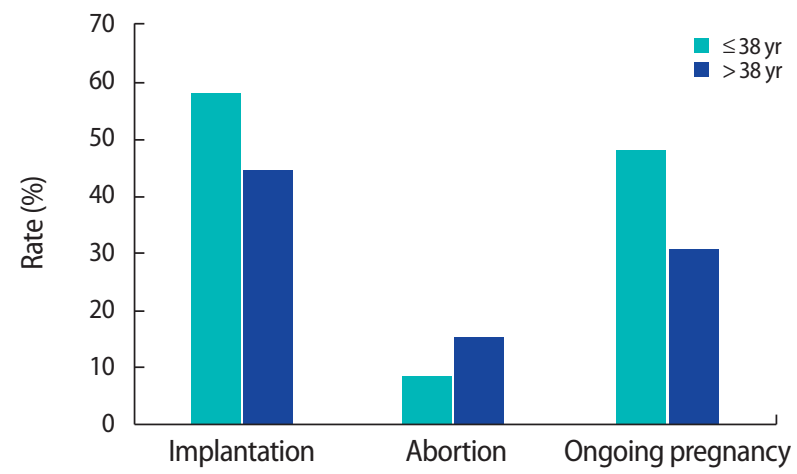

Figure 3. Comparisons of clinical outcomes between paternal age groups after adjusting for maternal age. The implantation and ongoing pregnancy rates were higher, and the abortion rate was lower, in the younger paternal age group, but without statistical significance. Therefore, paternal age did not have a significant influence on clinical outcomes.

depending on the morphological grade of the blastocysts.

Figure 3 demonstrates the differences between the paternal age groups in clinical outcomes after adjusting for maternal age. The implantation rate (paternal age $\leq 38$ vs. $>38$ years: $57.4 \%$ vs. $43.5 \%$, $p=0.061)$ and ongoing pregnancy rate ( $47.5 \%$ vs. $30.4 \%, p=0.275)$ were higher for the younger age group, but this trend was not statistically significant. The abortion rate $(8.2 \%$ vs. $15.2 \%, p=0.798)$ was also higher in the older paternal age group, but without significance, meaning that paternal age did not significantly influence the clinical outcomes of IVF.

\section{Discussion}

This study aimed to evaluate the potential effects of increasing paternal age on embryo aneuploidy and clinical outcomes. The statistical analysis demonstrated that maternal age had a major effect on the euploidy of the embryo, but paternal age did not have a significant impact. The relationships of paternal age with the euploidy rate and clinical outcomes such as the implantation, ongoing pregnancy, and abortion rates were not significant. To summarize, paternal age did not show a significant influence on these pregnancy-related outcomes when IVF with PGS was utilized.

Some data supporting the hypothesis that older parental age affects fertility potential have been reported. Several studies have found that older paternal age led to deteriorated sperm parameters such as reduced semen volume, sperm concentration, motility, and normal morphology $[13,14]$. These trends may be due to the increasing incidence of urogenital infections with age, as well as the accumulation of toxins and vascular diseases that alter the anatomy and function of the seminal pathway [15]. Furthermore, correlations between older paternal age and DNA damage have demonstrated that increased paternal age is associated with higher rates of mutations [16-18].

However, the present study demonstrated that the embryo and clinical outcomes were not significantly different between the two paternal age groups. A recent study also reported that several paternal variables, including age, body mass index, and sperm parameters, had negligible effects on the clinical outcomes of donor oocyte IVF [19]. Moreover, the findings of this study are in accordance with previous research showing that poor sperm parameters did not impact pregnancy outcomes when ICSI was utilized, since ICSI minimized the detrimental effect of the lowest-quality semen samples on fertilization [20-22].

Two prior studies utilized PGS to evaluate the effect of increased paternal age on embryo ploidy. The first study was a retrospective review of 286 embryos from 32 IVF cycles with ICSI and donated oocytes [23]. The researchers used 12-chromosome fluorescence in situ hybridization (FISH) analysis and found significantly higher rates of DNA damage in sperm and aneuploidy in embryos of men older than 50. However, the FISH method is known to be less accurate for detecting chromosomal abnormalities, whereas aCGH is a more developed and sensitive technology [24]. Therefore, the study by Garcia-Ferreyra et al. [23] may have had a greater number of falsely diagnosed euploid embryos, leading to different results from those of the present study. The second study by Tiegs et al. [25] was a recent retrospective study utilizing aCGH that showed similar results to this study. The study investigated 573 single thawed euploid embryo transfers from 473 patients undergoing oocyte donor and autologous IVF cycles, and concluded that there was no significant difference in pregnancy outcomes across all paternal age categories after euploid embryo transfer.

The major limitation of this study is its retrospective nature. The numbers of study subjects in each of the four groups were not even, and the total number of participants was small due to the short follow-up period. A future study with a longer follow-up period and larger study population will be helpful for confirming this study's findings. Additionally, it would be advisable to have longer follow-up periods to determine whether older paternal age is associated with more congenital anomalies or developmental disorders. Such a longterm evaluation was beyond the scope of this study, and future welldesigned prospective studies should investigate these issues. In conclusion, paternal age did not significantly influence the embryo euploidy rate. Clinical outcomes such as the pregnancy and abortion rates were also not significantly affected.

\section{Conflict of interest}

No potential conflict of interest relevant to this article was reported. 


\section{ORCID}

Min Kyoung Kim https://orcid.org/0000-0002-1753-2674

Jae Kyun Park https://orcid.org/0000-0002-5333-4990

Yunmi Jeon https://orcid.org/0000-0003-2751-8718

Su Hee Seok https://orcid.org/0000-0003-3595-8983

Eun Mi Chang https://orcid.org/0000-0001-9256-5057

Woo Sik Lee https://orcid.org/0000-0002-2329-1774

\section{Author contributions}

Conceptualization:WSL, JKP. Data curation:YJ, SHS, EMC. Methodology: JKP, MKK. Writing - original draft: MKK. Writing - review \& editing: MKK, WSL.

\section{References}

1. Ziebe S, Loft A, Petersen JH, Andersen AG, Lindenberg S, Petersen $\mathrm{K}$, et al. Embryo quality and developmental potential is compromised by age. Acta Obstet Gynecol Scand 2001;80:169-74.

2. Szamatowicz M, Grochowski D. Fertility and infertility in aging women. Gynecol Endocrinol 1998;12:407-13.

3. Pantos K, Athanasiou V, Stefanidis K, Stavrou D, Vaxevanoglou T, Chronopoulou M. Influence of advanced age on the blastocyst development rate and pregnancy rate in assisted reproductive technology. Fertil Steril 1999;71:1144-6.

4. Khandwala YS, Zhang CA, Lu Y, Eisenberg ML. The age of fathers in the USA is rising: an analysis of 168867480 births from 1972 to 2015. Hum Reprod 2017;32:2110-6.

5. Dain L, Auslander R, Dirnfeld M. The effect of paternal age on assisted reproduction outcome. Fertil Steril 2011;95:1-8.

6. Sagi-Dain L, Sagi S, Dirnfeld M. Effect of paternal age on reproductive outcomes in oocyte donation model: a systematic review. Fertil Steril 2015;104:857-65.

7. Robertshaw I, Khoury J, Abdallah ME, Warikoo P, Hofmann GE. The effect of paternal age on outcome in assisted reproductive technology using the ovum donation model. Reprod Sci 2014; 21:590-3.

8. Kaarouch I, Bouamoud N, Madkour A, Louanjli N, Saadani B, Assou $S$, et al. Paternal age: negative impact on sperm genome decays and IVF outcomes after 40 years. Mol Reprod Dev 2018;85: 271-80.

9. Campos I, Gomez E, Fernandez-Valencia AL, Landeras J, Gonzalez $R$, Coy $P$, et al. Effects of men and recipients' age on the reproductive outcome of an oocyte donation program. J Assist Reprod Genet 2008;25:445-52.

10. Neri QV, Monahan D, Rosenwaks Z, Palermo GD. Intracytoplas- mic sperm injection: technical aspects. In: Gardner DK, Weissman A, Howles CM, Shoham Z, editors. Textbook of assisted reproductive techniques. 4th ed. Boca Raton: Informa Healthcare; 2012. p. 172-82.

11. Silverberg KM, Turner T. Evaluation of sperm. In: Gardner DK, Weissman A, Howles CM, Shoham Z, editors. Textbook of assisted reproductive techniques. 4th ed. Boca Raton: Informa Healthcare; 2012. p. 48-59.

12. Gardner DK, Schoolcraft WB. In vitro culture of human blastocysts. In: Jansen R, Mortimer D, editors. Towards reproductive certainty: fertility and genetics beyond 1999. Carnforth: Parthenon Publishing; 1999. p. 378-88.

13. Eskenazi B, Wyrobek AJ, Sloter E, Kidd SA, Moore L, Young S, et al. The association of age and semen quality in healthy men. Hum Reprod 2003;18:447-54.

14. Kidd SA, Eskenazi B, Wyrobek AJ. Effects of male age on semen quality and fertility: a review of the literature. Fertil Steril 2001; 75:237-48.

15. Kuhnert B, Nieschlag E. Reproductive functions of the ageing male. Hum Reprod Update 2004;10:327-39.

16. Vagnini L, Baruffi RL, Mauri AL, Petersen CG, Massaro FC, Pontes $A$, et al. The effects of male age on sperm DNA damage in an infertile population. Reprod Biomed Online 2007;15:514-9.

17. Schmid TE, Eskenazi B, Baumgartner A, Marchetti F, Young S, Weldon $R$, et al. The effects of male age on sperm DNA damage in healthy non-smokers. Hum Reprod 2007;22:180-7.

18. Wyrobek AJ, Eskenazi B, Young S, Arnheim N, Tiemann-Boege I, Jabs EW, et al. Advancing age has differential effects on DNA damage, chromatin integrity, gene mutations, and aneuploidies in sperm. Proc Natl Acad Sci U S A 2006;103:9601-6.

19. Capelouto SM, Nagy ZP, Shapiro DB, Archer SR, Ellis DP, Smith AK, et al. Impact of male partner characteristics and semen parameters on in vitro fertilization and obstetric outcomes in a frozen oocyte donor model. Fertil Steril 2018;110:859-69.

20. Begueria R, Garcia D, Obradors A, Poisot F, Vassena R, Vernaeve V. Paternal age and assisted reproductive outcomes in ICSI donor oocytes: is there an effect of older fathers? Hum Reprod 2014; 29:2114-22.

21. Ho J, Noel M, Kao CN, Mok-Lin E, Cedars MI, Huddleston HG. Semen parameters and paternal age do not affect fertilization rate or pregnancy outcomes in oocyte recipient cycles. Fertil Steril 2015;103(2 Suppl):e23-4.

22. Barros B, Domingues TS, Belo AS, Mazetto R, Aquino AP, Motta EL. Influence of seminal quality in donor egg IVF program using vitrified oocytes. Fertil Steril 2015;104(3 Suppl):e195.

23. Garcia-Ferreyra J, Luna D, Villegas L, Romero R, Zavala P, Hilario R, et al. High aneuploidy rates observed in embryos derived from 
donated oocytes are related to male aging and high percentages of sperm DNA fragmentation. Clin Med Insights Reprod Health 2015;9:21-7.

24. Gutierrez-Mateo C, Colls P, Sanchez-Garcia J, Escudero T, Prates R, Ketterson $\mathrm{K}$, et al. Validation of microarray comparative genomic hybridization for comprehensive chromosome analysis of embryos. Fertil Steril 2011;95:953-8.

25. Tiegs AW, Sachdev NM, Grifo JA, McCulloh DH, Licciardi F. Paternal age is not associated with pregnancy outcomes after single thawed euploid blastocyst transfer. Reprod Sci 2017;24:1319-24. 